\title{
INFLUÊNCIA DO MÉTODO DE SAPONIFICAÇÃO DE ÓLEO VEGETAL AMAZÔNICO NA FLOTABILIDADE DA APATITA
}

\author{
P. S. OLIVEIRA, A. C. OLIVEIRA, J. K. S. PINTO, D. S. COSTA e R. S. PAIVA \\ Universidade Federal do Sul e Sudeste do Pará \\ eng.priscila.oliveira@hotmail.com; amandaminas09@gmail.com; jkerina2006@yahoo.com.br; \\ denilson@ufpa.br; regisabo@ufpa.br
}

Artigo submetido em novembro/2013 e aceito em julho/2014

DOI: http://dx.doi.org/10.15628/holos.2014.1819

\section{RESUMO}

Pesquisas demonstram a viabilidade técnica da utilização de sais de ácidos graxos, obtidos a partir da hidrólise alcalina de óleos provenientes de espécies vegetais amazônicas como coletores em flotação de minérios fosfáticos. No entanto, a necessidade de torná-los solúveis em água através da reação de saponificação, que é feita em alta temperatura e tempo elevado de reação, dificulta a sua aplicação a nível industrial. Nesse sentido, o objetivo principal deste trabalho é avaliar diferentes métodos de saponificação do óleo vegetal extraído da polpa de buriti (Mauritia flexuosa), oriundo da floresta amazônica, analisando a influência do método na flotabilidade da apatita. Nas reações de saponificação variou-se o tempo e a temperatura de reação, a proporção água/álcool e proporção óleo/ $\mathrm{NaOH}$. O critério de flotabilidade utilizado foi a microflotação em tubo de Hallimond modificado, com emprego de extensor da altura do aparato para evitar o arraste hidrodinâmico. Os resultados de flotabilidade obtidos apontam como as melhores condições: tempo máximo de reação de 20 minutos, proporção óleo/ $\mathrm{NaOH}$ de $1: 0,3$, proporção álcool/água de 1:0 e temperatura de reação de $55^{\circ} \mathrm{C}$.

PALAVRAS-CHAVE: hidrólise alcalina, coletores, microflotação.

\section{INFLUENCE OF THE SAPONIFICATION METHOD OF AMAZONIAN OIL ON THE FLOATABILITY OF APATITE}

\begin{abstract}
Investigations demonstrated the technical viability of the use of fatty acid salts, obtained via the alkaline hydrolysis of oils from Amazonian plant species, as collectors in phosphate ore flotation. However, the need to render them water soluble by saponification reaction, which is performed at high temperature and long reaction time, hampers their industrial use. Thus, the main objective of this study is to evaluate different methods of saponification of the vegetable oil extracted from the buriti pulp, originated from the Amazon rainforest,
\end{abstract}

analyzing the influence of the method in the floatability of apatite. In the saponification reactions, temperature and time of reaction, water/alcohol ratios, and oil/hydroxide ratios were varied. The floatability criterion used was the microflotation test in modified Hallimond tube with height extension to avoid entrainment. The floatability results suggested a saponification method with 20 minutes reaction time, oil/hydroxide ratio of $1: 0.3$, alcohol/water ratio of $1: 0$, and temperature of reaction of $55^{\circ} \mathrm{C}$.

KEYWORDS: alkaline hydrolysis; collectors; microflotation. 


\section{INTRODUÇÃO}

Embora o setor mineral constitua um setor de grande importância para a sociedade e economia de qualquer país, é a atividade menos aceita dentro do novo conceito de desenvolvimento sustentável devido à sua natureza. Então, faz-se necessário uma reavaliação dos insumos e processos utilizados pela indústria mineral, de modo a adequá-los aos novos padrões exigidos.

A região amazônica detém quantidade considerável de espécies vegetais cujas propriedades as qualificam como fortes candidatas a serem utilizadas como reagentes na flotação de minérios. Essas espécies apresentam como características principais a alta disponibilidade e o fato de ainda não serem aproveitadas industrialmente em quantidade significativa. Além disso, estes insumos são provenientes de fontes renováveis e biodegradáveis, o que evitaria problemas de agressão ambiental e se adapta ao novo cenário buscado pela indústria mineral. A utilização desses insumos também agregaria valor às espécies amazônicas, contribuindo, assim, com o desenvolvimento socioeconômico da região.

Pesquisas já comprovaram a viabilidade técnica da utilização dos óleos amazônicos como coletores de minérios fosfáticos (COSTA, 2012). Contudo, para a utilização desses insumos como coletores no processo de flotação, é necessário torná-los solúveis em água através da reação de saponificação, que é feita em alta temperatura e tempo elevado de reação. Além dos gastos com energia, os custos associados aos reagentes empregados nessa técnica, dificultam o seu uso industrial. Nesse sentido, é importante analisar o efeito da variação dos parâmetros utilizados na reação de saponificação, de modo que pela redução de gastos com reagentes e energia, haja a viabilidade econômica do emprego destes como coletores.

Assim, este trabalho teve como objetivo principal avaliar diferentes métodos de saponificação do óleo vegetal extraído da polpa de buriti (Mauritia flexuosa), oriundo da floresta amazônica, analisando a influência do método na flotabilidade da apatita.

\section{METODOLOGIA}

\section{1 Óleo vegetal}

Foi utilizado o óleo extraído da polpa de buriti (Mauritia flexuosa). A composição em ácidos graxos dessa espécie amazônica foi determinada através de cromatografia gasosa (CG) realizada no laboratório de cromatografia do Departamento de Química da Universidade Federal de Minas Gerais. A composição em ácidos graxos está mostrada na Tabela 1.

Tabela 1 - Composição em Ácidos Graxos do Óleo de Buriti.

\begin{tabular}{|c|c|c|c|c|c|c|}
\hline \multicolumn{7}{|c|}{ ÁCIDOS } \\
\hline LÁURICO & MIRÍSTICO & PALMÍTICO & ESTEÁRICO & OLEICO & LINOLEICO & LINOLÊNICO \\
\hline- & - & 17,44 & 2,17 & 70,55 & 7,03 & 1,08 \\
\hline
\end{tabular}

Fonte - Laboratório de Cromatografia do Departamento de Química da UFMG. 


\subsection{Hidrólise alcalina}

Para a hidrólise alcalina (saponificação) foram utilizados dois gramas do óleo de buriti e um sistema de manta aquecedora e refluxo. Os parâmetros analisados foram: tempo e temperatura de reação, proporção óleo/ $\mathrm{NaOH}$, proporção álcool/água. Neste trabalho foram utilizados álcool etílico (etanol) e água destilada.

Para a análise da influência do tempo na flotabilidade da apatita, fixou-se a temperatura de reação em aproximadamente $75^{\circ} \mathrm{C}$ e as proporções óleo/ $\mathrm{NaOH}$ e álcool/água em 1:0,15 e 1:0 (100\% de álcool), respectivamente. Esta mistura foi colocada em um balão e aquecida em tempos variáveis de $20,40,60$ e 80 minutos.

Para verificar a influência da proporção óleo/ $\mathrm{NaOH}$, repetiu-se os parâmetros temperatura de reação e proporção álcool/água utilizados anteriormente, e fixou-se o tempo de reação em 20 minutos. As proporções avaliadas foram 1:0,15; 1:0,3; e 1:0,5.

Para avaliar a influência da proporção álcool/água na flotabilidade do mineral, definiu-se a proporção óleo/ $\mathrm{NaOH}$ em 1:0,3 e o tempo e a temperatura de reação em 20 minutos e aproximadamente $75^{\circ} \mathrm{C}$, respectivamente. Os valores avaliados foram 1:1, 3:1 e 1:0.

Para analisar a influência da temperatura de reação, utilizou-se os parâmetros 20 minutos de reação, proporção 1:0,3 de óleo/ $\mathrm{NaOH}$ e proporção álcool/água de 1:0. Variou-se a temperatura de reação em $27,35,55$ e $75^{\circ} \mathrm{C}$.

\subsection{Amostra mineral}

O mineral utilizado nos ensaios de flotabilidade em tubo de Hallimond foi apatita. Na Figura 1 e Tabela II visualizam-se os resultados obtidos por difração de raios-X e fluorescência de raios-X, respectivamente, para a amostra de apatita.

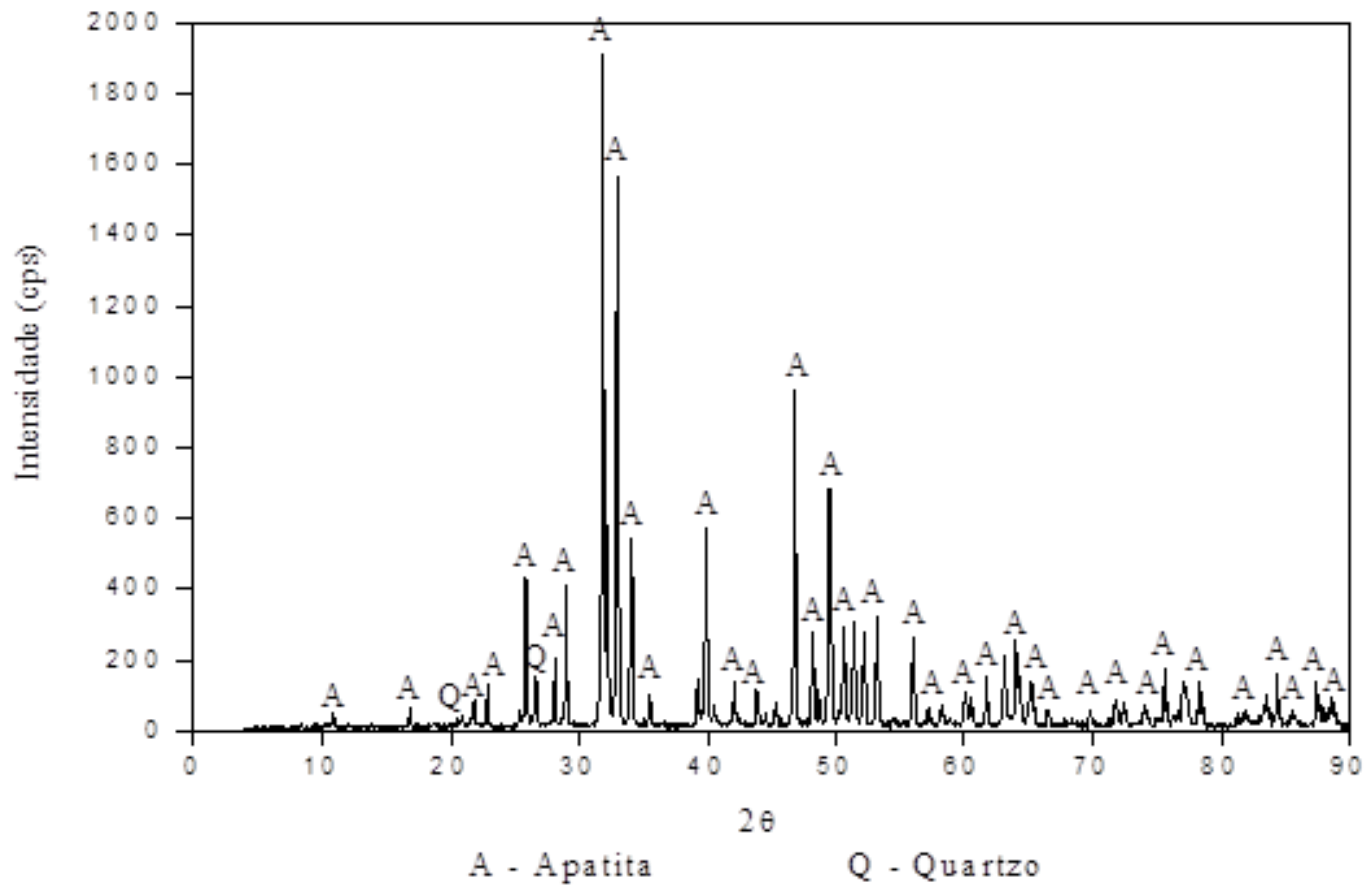

Figura 1 - Difratograma da mostra de apatita. 
Tabela 2 - Análise da Amostra de Apatita por Fluorescência de Raios-X (em \%)

\begin{tabular}{|c|c|c|c|c|c|c|c|c|c|c|c|c|}
\hline $\mathrm{CaO}$ & $\mathrm{CO}_{2}$ & $\mathrm{P}_{2} \mathrm{O}_{5}$ & MgO & $\mathrm{SiO}_{2}$ & $\mathrm{Al}_{2} \mathrm{O}_{3}$ & $\mathrm{MnO}$ & $\mathrm{PbO}$ & SrO & $\mathrm{Fe}_{2} \mathrm{O}_{3}$ & $\mathrm{Y}_{2} \mathrm{O}_{3}$ & $\mathrm{Cl}$ & Th \\
\hline 58,16 & - & 26,14 & 5,90 & 5,75 & 3,09 & 0,03 & 0,02 & 0,04 & 0,10 & 0,02 & 0,55 & 0,19 \\
\hline
\end{tabular}

Fonte - Departamento de Química da UFMG.

Pela Figura 1, conclui-se que a amostra mineral usada neste trabalho é predominantemente composta por apatita, com pequena quantidade do mineral quartzo. Esta conclusão é ratificada pelos resultados obtidos através da fluorescência de raios-X (Tabela II).

Portanto, de acordo com os resultados apresentados, o mineral usado neste estudo está com pureza aceitável para a realização dos ensaios de flotabilidade propostos.

\subsection{Microflotação em tubo de Hallimond modificado}

Os ensaios de microflotação da apatita foram realizados em duplicata utilizando tubo de Hallimond modificado com emprego de extensor de altura para evitar o arraste excessivo. As condições dos ensaios foram: $\mathrm{pH} 10,0$ (aproximadamente); vazão de ar de $40,0 \mathrm{~cm}^{3} / \mathrm{min}$; e tempos de condicionamento e flotação de 4 e 1 minuto, respectivamente. A massa do mineral foi de $1 \mathrm{~g}$, com granulometria entre 300 e $106 \mu \mathrm{m}$. As concentrações testadas foram de 0,$1 ; 0,2 ; 0,5 ; 1,0 ; 2,0$; 5,0; 7,5; e 10,0 mg/L. A flotabilidade da apatita (em \%) foi determinada a partir da relação entre a massa flotada e a massa total da amostra, descontando o arraste.

\section{RESULTADOS E DISCUSSÃO}

\subsection{Análise do tempo de reação}

A Figura 2 apresenta os resultados de flotabilidade da apatita obtidos variando o tempo de hidrólise alcalina (saponificação) e a concentração de coletor.

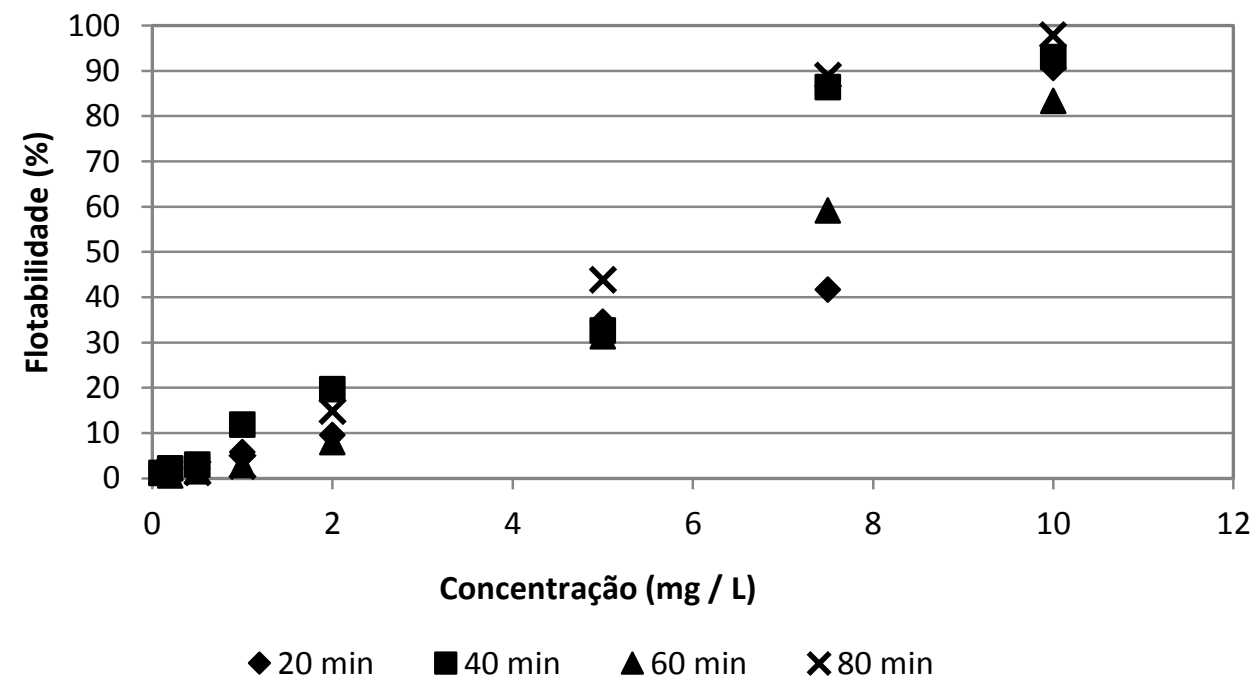

Figura 2 - Flotabilidade da apatita em função do tempo de saponificação. 
Pela Figura 2, verifica-se que o tempo de 80 minutos foi o que apresentou as melhores recuperações nas concentrações de 5,0, 7,5 e 10,0 mg/L, não variando, entretanto, consideravelmente em relação aos outros tempos de saponificação. Percebe-se, então, que a variação do tempo acima de 20 minutos de reação não é um fator determinante para a flotabilidade da apatita com o uso do coletor a partir do óleo de buriti.

\subsection{Análise da proporção óleo/ $\mathrm{NaOH}$}

Na Figura 3 são mostradas as recuperações de apatita com coletor saponificado variando a proporção óleo/NaOH e a concentração de coletor.

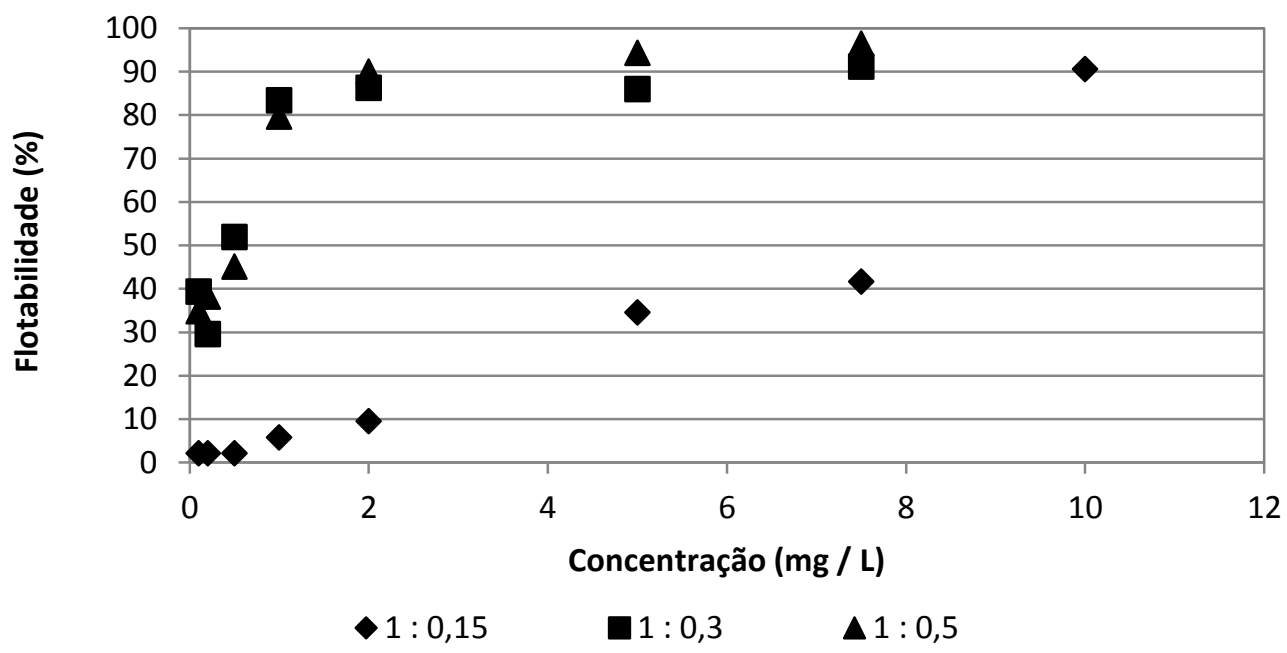

Figura 3 - Flotabilidade da apatita em função da proporção óleo/NaOH.

A partir da Figura 3, observa-se o aumento da eficiência do coletor com o aumento da quantidade de $\mathrm{NaOH}$ empregada durante a saponificação. Para as proporções óleo/ $\mathrm{NaOH}$ de 1:0,3 e 1:0,5, as baixas concentrações de coletor já apresentaram altas recuperações em relação à proporção 1:0,15. Para as maiores quantidades de hidróxido, recuperações de cerca de $90 \%$ foram observadas a concentração de $2 \mathrm{mg} / \mathrm{L}$ de coletor, enquanto que para a proporção de 1:0,15, uma recuperação de aproximadamente $90 \%$ ocorreu somente à concentração de 10,0 mg/L.

Observa-se também pela Figura 3, que não houve diferença significativa de desempenho do coletor entre as proporções óleo/ $\mathrm{NaOH}$ de 1:0,3 e 1:0,5, evidenciando que para quantidades de hidróxido de sódio acima de $30 \%$ da quantidade de óleo (proporção 1:0,3) não acarreta ganhos significativos de recuperação da apatita.

$\mathrm{O}$ aumento do poder de coleta do reagente em função do aumento da quantidade de $\mathrm{NaOH}$ deve-se ao fato de que, em uma reação de saponificação, o excesso do hidróxido alcalino em solução favorece a formação do produto e este, segundo Oliveira (2005), atua como catalisador da reação, favorecendo ainda mais a conversão do óleo em sais de ácidos graxos (coletor).

\subsection{Análise da proporção álcool/água}

A Figura 4 demonstra a maior eficiência do coletor obtido a partir da saponificação utilizando uma solução de $\mathrm{NaOH}$ 100\% alcoólica (proporção 1:0). No entanto, para concentrações 
maiores (7,5 e 10,0 mg/L) o coletor cuja reação de saponificação utilizou uma proporção de 75\% de álcool e 25\% de água (3:1) apresentou maiores recuperações. O coletor produzido com uma solução de hidróxido com $50 \%$ de água e $50 \%$ de álcool (proporção 1:1), apresentou recuperação do mineral apenas a partir da concentração de $2,0 \mathrm{mg} / \mathrm{L}$, sendo estas recuperações ainda muito inferiores às obtidas nos outros métodos.

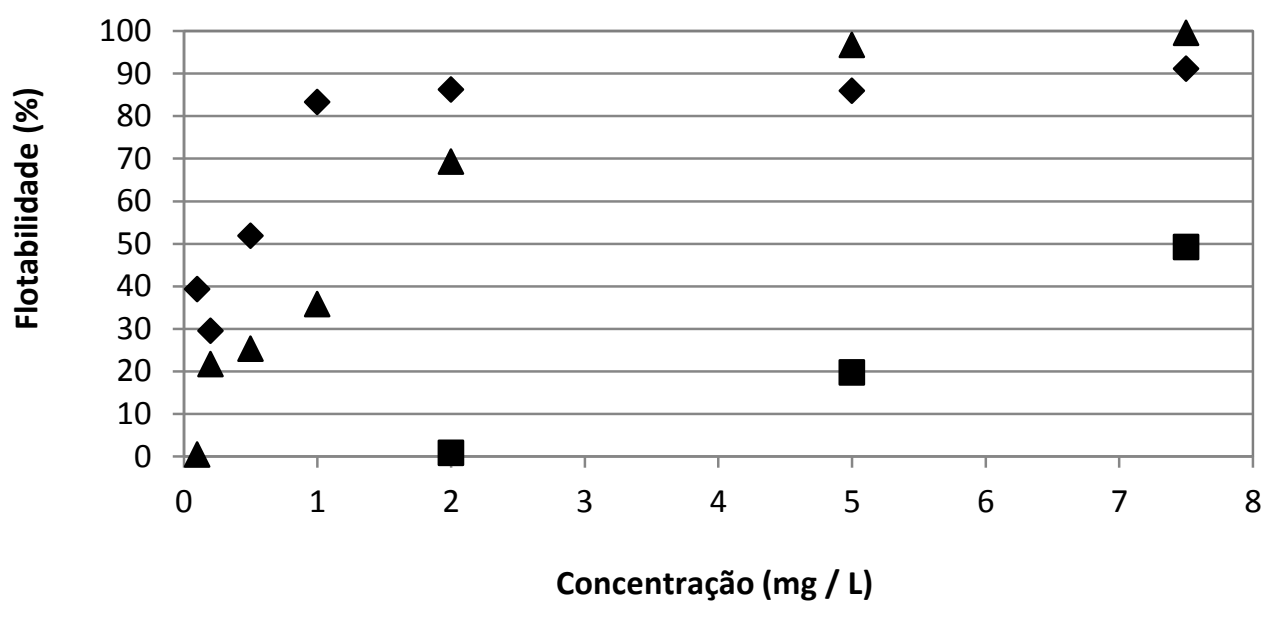

• $100 \%$ álcool $\quad \Delta 3: 1 \quad \square 1: 1$

Figura 4 - Flotabilidade da apatita em função da proporção água/álcool.

Azevedo et al. (1994), citados por Oliveira (2005), realizaram estudo do desempenho dos processos de saponificação do óleo de arroz e do óleo de rícino, utilizando seis maneiras de desenvolver a reação: 1 - com água e $\mathrm{NaOH}$ a $65^{\circ} \mathrm{C} ; 2$ - com álcool etílico, $\mathrm{NaOH}$ e temperatura elevada sob refluxo; 3 - com água, $\mathrm{NaOH}$ e sabão a $90^{\circ} \mathrm{C} ; 4$ - com água, excesso de $\mathrm{NaOH}$ e sabão a $90{ }^{\circ} \mathrm{C} ; 5-$ com álcool, água e $\mathrm{NaOH}$ a $90^{\circ} \mathrm{C} ; 6$ - com água e $\mathrm{NaOH}$ em temperatura elevada sob refluxo. Eles concluíram que o método "2" de saponificação apresentou melhor desempenho.

\subsection{Análise da temperatura de reação}

A Figura 5 apresenta os resultados obtidos para o coletor a partir do óleo de buriti, variando a temperatura de reação.

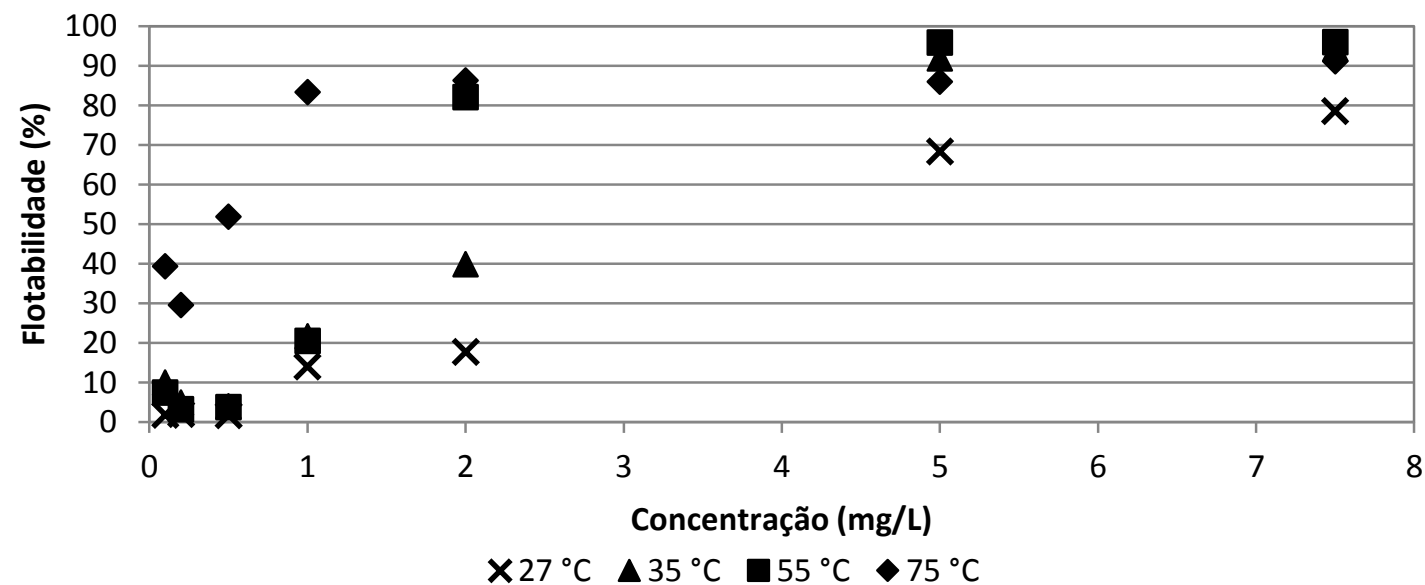

Figura 5 - Flotabilidade da apatita em função da temperatura de reação 
A Figura 5 mostra que, para pequenas concentrações $(0,1 \mathrm{mg} / \mathrm{L}-1,0 \mathrm{mg} / \mathrm{L})$, o método de saponificação que emprega uma temperatura de reação de $75{ }^{\circ} \mathrm{C}$ é o mais eficiente dentre os métodos estudados. Entretanto, para a concentração de coletor de 2,0 mg/L, a temperatura de 55 ${ }^{\circ} \mathrm{C}$ já torna-se eficiente para a conversão; e para concentrações ainda superiores $(5,0 \mathrm{mg} / \mathrm{L}$ e 7,5 $\mathrm{mg} / \mathrm{L})$, todos os métodos são eficientes. Apenas o método que utiliza a temperatura ambiente apresentou uma recuperação um pouco inferior.

Assim, tem-se que para o óleo de buriti, tende-se a obter um método de saponificação eficiente à temperatura de $55^{\circ} \mathrm{C}$, não sendo necessário maior emprego de energia.

\section{CONCLUSÕES}

A variação do tempo acima de 20 minutos de reação não é um fator determinante para a flotabilidade da apatita com o uso do coletor a partir do óleo de buriti.

Quantidades de hidróxido de sódio acima de 30\% da quantidade de óleo (proporção 1:0,3) não acarretam ganhos significativos de recuperação da apatita.

Embora o método de saponificação que empregou a proporção álcool/água de 3:1 tenha sido o que apresentou maiores recuperações em concentrações maiores de coletor (acima de 5,0 $\mathrm{mg} / \mathrm{L}$ ), o método que utiliza a solução $100 \%$ alcoólica foi a que apresentou as melhores recuperações em menores concentrações de coletor.

Tende-se a obter um método de saponificação eficiente à temperatura de $55^{\circ} \mathrm{C}$.

\section{AGRADECIMENTOS}

Os autores agradecem ao Instituto Tecnológico Vale e à FAPESPA pelo suporte financeiro.

\section{REFERÊNCIAS BIBLIOGRÁFICAS}

1. COSTA, D.S. Uso de óleos amazônicos na flotação de minérios fosfáticos. Tese de Doutorado. Programa de Pós-Graduação em Engenharia Metalúrgica, Materiais e de Minas, Universidade Federal de Minas Gerais, 176 p., 2012.

2. OLIVEIRA, J.A. Grau de saponificação de óleos vegetais na flotação seletiva de apatita de mineral carbonatítico. Dissertação de Mestrado. Departamento de Engenharia de Minas, Universidade Federal de Ouro Preto, 187 p., 2005. 